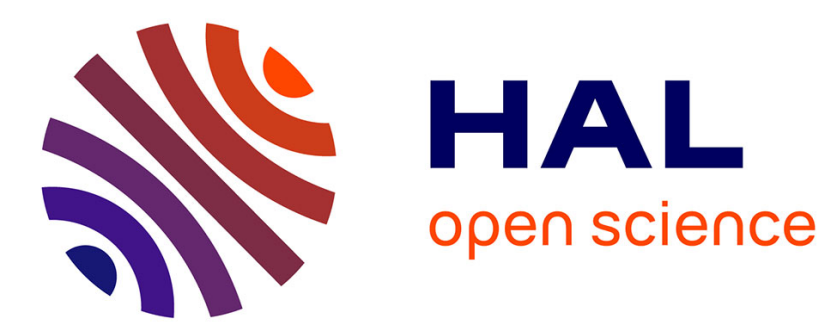

\title{
Strain partitioning in an accretionary wedge, in oblique convergence: analogue modelling
}

\author{
Alexandra Martinez, Jacques Malavieille, S. Lallemand, Jean-Yves Collot
}

\section{To cite this version:}

Alexandra Martinez, Jacques Malavieille, S. Lallemand, Jean-Yves Collot. Strain partitioning in an accretionary wedge, in oblique convergence: analogue modelling. Bulletin de la Société Géologique de France, 2002, 173 (1), pp.17-24 [428]. 10.2113/173.1.17 . hal-00406707

\section{HAL Id: hal-00406707 https://hal.science/hal-00406707}

Submitted on 8 Nov 2021

HAL is a multi-disciplinary open access archive for the deposit and dissemination of scientific research documents, whether they are published or not. The documents may come from teaching and research institutions in France or abroad, or from public or private research centers.
L'archive ouverte pluridisciplinaire HAL, est destinée au dépôt et à la diffusion de documents scientifiques de niveau recherche, publiés ou non, émanant des établissements d'enseignement et de recherche français ou étrangers, des laboratoires publics ou privés. 


\title{
Partition de la déformation dans un prisme d'accrétion sédimentaire en convergence oblique : approche expérimentale
}

\author{
ALEXANDRA MARTINEZ ${ }^{1}$, JACques MALAVIEILLE ${ }^{1}$, SERge LALLEMAND ${ }^{1}$ et JeAN-Yves COLlOT ${ }^{2}$
}

\begin{abstract}
Mots clés. - Convergence oblique, Partition du mouvement, Vecteur glissement, Prisme d'accrétion, Décrochement, Modélisation analogique, Prisme de Coulomb.

Résumé. - Au niveau des zones de subduction oblique, la partition du mouvement se traduit souvent par une déformation importante caractérisée par la coexistence de chevauchements avec de grandes failles décrochantes. Dans un prisme d'accrétion, des paramètres comme l'obliquité de la convergence et la friction à la base du prisme peuvent influencer cette partition. Ces paramètres sont testés à l'aide de modèles analogiques pour deux géométries de butées rigides. Les expériences montrent que la pente de stabilité du prisme reste constante et proche de $\alpha_{\text {coulomb }}$ pour des obliquités de convergence variables. L'analyse des résultats suggère que l'équation sur la stabilité des prismes de Coulomb s'appliquerait seulement dans la direction de convergence et donc que les paramètres déduits de la théorie seraient uniquement valables dans cette direction. On constate également que le taux de partition augmente avec l'obliquité de convergence et que la partition se produit quelle que soit la friction basale. La morphologie de la structure est modifiée au-delà d'une certaine valeur d'obliquité. Dès lors, un faisceau cisaillant s'initie.

Nos modèles montrent la présence de structures obliques associées au décrochement et localisées à l'aplomb de la discontinuité de vitesse. Des structures semblables à celles-ci ont été observées en Nouvelle-Zélande au niveau du prisme d'Hikurangi.
\end{abstract}

\section{Strain partitioning in an accretionary wedge, in oblique convergence : analogue modelling}

Key words. - Oblique convergence, Strain partitioning, Slip vector, Accretionary wedge, Strike-slip fault, Analog model, Coulomb wedge.

Abstract. - In subduction zones undergoing oblique convergence, strain partitioning is often expressed by an important deformation inducing strike-slip faulting. In accretionary wedges, parameters such as obliquity of the convergence and friction at the bottom of the wedge play an important role in the strain partitioning. The impact of these parameters is studied using sandbox experiments. Two backstop geometries have been designed to account for different geological settings. These experiments show that the wedge taper remains constant and close to $\alpha_{\text {coulomb }}$ for variable obliquities. Measurements of critical tapers on the models suggest that the Coulomb wedge theory cannot be simply applied to determine parameters on wedges developed under oblique convergence. Parameters deduced from this theory are valid only when measured in the direction parallel to the convergence. In addition, the partition degree increases with the obliquity of the convergence, and strain partitioning occurs independently of the basal friction. We remark that the model morphology changes when an obliquity value, mainly, is exceeded. A transcurrent structure develops. The models show that oblique structures located above the velocity discontinuity are associated with strike-slip faults. Similar structures have been observed within the Hikurangi accretionary wedge (New Zealand).

Introduction. - In subduction zones, for high values of the convergence obliquity $\gamma$, transcurrent faults are observed, parallel to the trench, on the continental plate. Such structures are present in Sumatra and the Philippines along the volcanic arc [Bellier and Sébrier, 1995 ; Malod et al., 1993 ; Barrier et al. 1991] or at the rear of the accretionary wedge for the South Ryukyu (Taiwan). These strike-slip faults are a consequence of strain partitioning. Partition is controlled by obliquity of convergence, basal friction and probably geometry of backstop. Using analog models, the influence of each parameter on strain partitioning, on the occurrence of transcurrent faults and its morphology are analyzed.

The strain partitioning can be quantified by its degree $\mathrm{K}_{\mathrm{v}}: K_{v}=\left(1-\frac{\psi}{\gamma}\right) \cdot 100$

It requires the knowledge of the slip vector, which is situated between the azimuth of the convergence and the perpendicular to the trench.

Experimental procedure. - The aim of our experiments is to better understand the effects of several parameters which determine the strain partitioning in accretionary wedges undergoing oblique convergence. For this purpose, a large-size table $(140 \mathrm{~cm} \times 250 \mathrm{~cm})$ covered with a plastic film moving towards the backstop is used (fig. 1). The basal friction of the wedge can change according to the different textures of the plastic films used. Various angles of convergence $\left(0^{\circ}, 20^{\circ}, 40^{\circ}\right.$ and $\left.60^{\circ}\right)$ are studied (fig. 1).

Two types of backstop geometries are designed. In the first case, the plastic sheet moves under a thin PVC plate resting at the base of the material, in order to localize the strain above the velocity discontinuity, at the tip of the plate. In the second case, the backstop presents a $4 \mathrm{~cm}$ high, low-friction vertical wall. The whole device is covered by $2 \mathrm{~cm}$

\footnotetext{
${ }^{1}$ Laboratoire de Géophysique, Tectonique et Sédimentologie, UMR 5573, cc. 60, Université Montpellier 2, 34095 Montpellier cedex 5.

2 Géosciences Azur, ORSTOM, B.P. 48, 06235, Villefranche s/mer cedex

Manuscrit déposé le 11 août 2000 ; accepté après révision le 9 juillet 2001.
} 
of dry sand. The sand satisfies a Coulomb failure envelope, with an internal friction angle of about $30^{\circ}$ and a very low cohesion. Colored markers perpendicular and parallel to the convergence direction, and perpendicular to the backstop, are spread on the surface of the sand. They allow recording of kinematics during the experiments.

During these experiments, the plastic film converges towards the backstop with a constant velocity. Pictures of the deforming model are taken perpendicularly to the surface, every $5 \mathrm{~cm}$ of shortening. Intersections of the colored lines are followed and recorded at every stage of shortening, in order to describe the deformation and determine the velocity field. Vs corresponds to the displacement-vector of these points. The knowledge of the kinematics allows to deduce (using geometric construction) the slip-vector $\mathrm{V}_{\mathrm{g}}$ and its obliquity $\psi$ relative to the normal to the trench.

The partition degree $\mathrm{K}_{\mathrm{V}}$ is a function of the obliquity of convergence vector and of the obliquity of slip vector (fig. 2). The critical taper is measured at the end of each experiment, perpendicularly to the backstop and parallely to the direction of convergence.

Results and discussion. - The morphology of the wedge suggests different structural domains : (a) a stable domain, (b) a zone of wrenching, (c) a wedge of imbricated thrusts. Above a value of the obliquity of convergence, the three domains become more distinct. Oblique structures develop in relation with the strike-slip fault. Their orientation compared with the orientation of the strike-slip fault is controlled by the geometry of the backstop (fig. 3). In the first type, these structures seem to be perpendicular to the convergence, in contrast with the second type where they tend to be parallel.

Physical models of accretionary wedges show that the critical taper increases with increasing basal friction [e.g., Malavieille et al., 1992]. Furthermore, the sand used in the experiments obeys Coulomb failure criterion. Davis et al. [1983] apply this criterion to convergent thrust-wedges. They determine a relationship between critical taper wedge $\left(\alpha_{\text {coulomb }}\right)$, dip $(\beta)$ of the subducting plate and basal friction $(\mu b)$ :

$$
\alpha_{\text {coulomb }}+\gamma=\frac{(\mu b+\beta)}{(1+K)}
$$

In our experiments, for both geometries of backstop, the slope measured perpendicularly to the trench increases with the obliquity of the convergence, whereas the slope measured parallel to the direction of the convergence remains constant. Its value is close to the slope deduced from relation 1 . The slope measured perpendicularly to the trench increases with increasing basal friction (fig. 4).

The partition degree $\mathrm{K}_{\mathrm{v}}$ depends on the relation between the convergence vector $\mathrm{V}_{\mathrm{c}}$ and the slip vector $\mathrm{V}_{\mathrm{g}}$. Each of them is defined by their obliquities which are respectively $\gamma$ and $\psi$. The partition degree $\mathrm{K}_{\mathrm{v}}$ can be deduced from the obliquity of the convergence vector relative to the slip vector, Liu et al. [1995]. See equation (1).

For both backstop geometries (fig. 5), Kv increases with the obliquity of the convergence $\gamma$. Moreover, the strain partitioning develops for $\gamma$ superior or equal to $20^{\circ}$. In the second type of experiments, the partition degree is homogeneous in the whole structure. It moves like a single block along a strike-slip fault located above the velocity discontinuity (fig. 6).

Studies on the critical taper allow Davis et al. [1983], to determine a relationship applied to a frontal convergence. In our experiments, the measurements of the critical taper of the accretionary wedge is equal to $\alpha_{\text {coulomb }}$ and remains constant in the direction of the convergence. The critical taper equation (2) seems to apply in the direction of convergence. So the measures taken in the direction of the convergence permit to obtain some correct parameters.

Using sand-silicone analogue models, Pinet and Cobbold [1992] show that a minimum angle of oblique convergence $\left(30^{\circ}\right)$ is required before strain partitioning occurs. Our models involving dry sand clearly reveal that strain partitioning :

1) increases with the obliquity of convergence,

2) may develop with low obliquities and

3) may also develop if basal friction is very low. From physical models, Chemenda et al. [2000] proposed that the strain partitioning at lithospheric scale may occur for a high interplate friction only. Thus, the rheology of the material probably has a strong influence on the critical value of the convergence obliquity beyond which strain partitioning may appear.

Comparison with natural examples. - To the south of the Ryukyu Trench, northeast Taiwan, bathymetric data highlight a dextral strike-slip fault at the rear of the accretionary wedge [Lallemand et al., 1999]. Seismic profiles analysis crossing the same area gives constraints on the shape of the Ryukyu arc basement. It ends with a several kilometers high subvertical basement wall [Font et al., 2001]. This vertical backstop favors strain partitioning and locates strike slip faulting. Our models also clearly outline the impact of backstop geometry on strain partitioning and on strain location.

In New-Zealand, along the Hikurangi margin, the Pacific plate meets the Australian plate at a very oblique convergence $\left(60^{\circ}\right)$. Between the Cook canyon $\left(175^{\circ} 00 \mathrm{E}\right.$ and $\left.176^{\circ} 45 \mathrm{E}\right)$, three crustal faults, having the same direction, are observed. The Palliser-Kaiwhata fault which outlines the edge of the continental margin is interpreted as a subvertical strike-slip fault. Close to the trench, two other faults are interpreted as reverse faults which may correspond to a strike slip oblique component. Sonar data reveals a lineament associated only with the Palliser-Kaiwhata fault trend [Barnes et al., 1998]. In our experiments, oblique structures associated to strike-slip faulting developed (backstop geometry 2, fig. 3d). These structures seem to be linked with strike-slip displacement of faults. Knowing the boundary conditions of the models and about the structural surface of the Hikurangi wedge, a similar configuration to our models could exist, with the possible presence of a velocity discontinuity above the Palliser-Kaiwhata fault.

The present study points out several results :

1) the critical taper of the wedge remains stable in the direction of the convergence when the obliquity increases. It is equal to the theoretical value determined by the Coulomb wedge theory;

2) the partition degree increases with increasing obliquity of the convergence, whatever the basal friction may be; 
3) different structural domains characterized by their morphology have been observed : a stable domain, a convergence zone of wrenching, and a wedge of imbricated thrusts;

4) oblique structures are associated to the strike slip fault zone.

\section{INTRODUCTION}

Dans les zones de subduction, pour certaines valeurs d'obliquité de la convergence, de grandes failles décrochantes parallèles au front de subduction sont fréquement observées à terre, comme par exemple à Sumatra ou aux Philippines le long de l'arc volcanique [Bellier et Sébrier, 1995 ; Bellier et al., 1991 ; Malod et al., 1993 ; Barrier et al., 1991] ou bien en mer à l'arrière du prisme d'accrétion, comme c'est le cas au sud des Ryukyus [Lallemand et al., 1999 ; Dominguez et al., 1998]. Ces décrochements sont une conséquence de la partition de la déformation, qui est contrôlée par l'obliquité de la convergence, le frottement basal et vraisemblablement par la géométrie de la butée rigide. Dans cette étude, l'influence de chacun de ces paramètres sur la partition du mouvement d'une part, le rôle de la géométrie de l'extrémité de la marge sur l'apparition de structures décrochantes et la morphologie d'autre part, sont analysés à l'aide d'une série de modèles analogiques. La partition du mouvement peut être quantifiée en calculant son taux : $\mathrm{K}_{\mathrm{v}}$. Son calcul nécessite de connaître le vecteur glissement de la subduction dont l'azimut est souvent compris entre celui de la convergence et celui de la normale à la fosse. $\mathrm{K}_{\mathrm{v}}$ est fonction de l'obliquité du vecteur convergence $\gamma$ et de l'obliquité du vecteur glissement $\psi$ :

$$
K_{v}=\left(1-\frac{\psi}{\gamma}\right) \cdot 100
$$

On examinera alors en détail la morphologie du prisme, l'évolution des pentes d'équilibre du prisme en convergence oblique, l'existence éventuelle d'un seuil d'obliquité au delà duquel la partition du mouvement se produirait, l'évolution du taux de partition avec l'augmentation de la friction basale $\mu \mathrm{b}$ et celle de l'obliquité de la convergence $\gamma$.

\section{PROCÉDURE}

Les modèles analogiques sont utilisés pour mieux comprendre l'effet de quelques paramètres qui influencent la partition de la déformation dans un prisme d'accrétion en convergence oblique. Nous utilisons une grande table $(140 \mathrm{~cm} \times 250 \mathrm{~cm})$ horizontale sur laquelle repose un film plastique qui se déplace vers une butée rigide (celle-ci représente l'extrémité indéformable de la marge continentale, zone contre laquelle les unités vont s'accréter). Les différentes textures du film plastique utilisé permettent de faire varier la friction à la base du prisme. La butée rigide pivotante permet d'imposer l'obliquité de la convergence. Des angles de $0^{\circ}, 20^{\circ}, 40^{\circ}$ et $60^{\circ}$ sont testés dans cette étude. Deux géométries simples de butée sont utilisées dans les modèles (fig. 1).

Dans le premier cas (géométrie 1), le film plastique est tiré sous une plaque fine ( $5 \mathrm{~mm}$ d'épaisseur). Il est disposé à la base du matériau afin de localiser la déformation à l'aplomb de la discontinuité de vitesse (DV) située à l'extrémité de la plaque. Dans le second cas (géométrie 2), la butée rigide présente un bord vertical de $4 \mathrm{~cm}$ avec une faible friction à l'interface, qui est localisé à l'aplomb de la discontinuité de vitesse. L'ensemble du dispositif est recouvert par une couche de sable de $2 \mathrm{~cm}$ d'épaisseur. Un protoprisme, dont la pente est définie en fonction de la friction imposée par le film, est mis en place contre la butée rigide. Dans la seconde série d'expériences, celui-ci est plus large afin de recouvrir entièrement la butée rigide qui est plus épaisse. Des marqueurs rectilignes colorés perpendiculaires et parallèles à la direction de convergence (espacés de $10 \mathrm{~cm}$ ) et perpendiculaires à la butée rigide (espacés de $40 \mathrm{~cm}$ ) sont déposés à la surface du sable.

Le sable sec utilisé pour construire le prisme possède les propriétés de friction satisfaisant la théorie de Coulomb [Dalhen et Suppe, 1984]. Ce sable non compacté a une densité de $1690 \mathrm{~kg} / \mathrm{m}^{3}$ et un coefficient de friction interne de 0,57 .

\section{Déroulement de l'expérience}

On fait converger le film plastique à vitesse constante vers la butée rigide à l'aide d'un moteur. Des photographies du modèle sont prises perpendiculairement à la surface, tous les $5 \mathrm{~cm}$ de raccourcissement. Lorsqu'elles sont préservées à la surface du prisme, les intersections entre les marqueurs colorés sont suivies. Leur déplacement est enregistré à tous les stades de raccourcissement, pour caractériser la déformation. Il est alors possible de décrire la cinématique des points déplacés à la surface du prisme. Vs correspond au vecteur déplacement de ces points. Lorsque la cinématique est connue sur $\mathrm{x} \mathrm{cm}$ de raccourcissement dans la direction de convergence, on déduit par construction géométrique entre $V_{s}$ et $V_{g}$ le vecteur glissement $V_{g}$ (direction relative du prisme qui se déplace vers la butée rigide) puis son obliquité $\psi$ par rapport à la normale à la fosse (fig. 2).

Le taux de partition $\mathrm{K}_{\mathrm{v}}$ est fonction de l'obliquité du vecteur convergence et de l'obliquité du vecteur glissement. Il est calculé pour plusieurs points dans chacune des expériences. Au stade final, on mesure les pentes du prisme perpendiculairement à la butée rigide (donc au front de déformation) $\alpha$ n et parallèlement à la direction de convergence $\alpha c$.

\section{RÉSULTATS}

Dans un premier temps, deux paramètres sont testés : $\gamma$ l'obliquité de la convergence et $\mu \mathrm{b}$ la friction basale, qui varient pour des géométries de butée rigide différentes. Dans la première série de modèles, la butée rigide est définie par une discontinuité de vitesse sans bord vertical, dans la seconde série elle présentera un bord vertical.

Une morphologie commune à la plupart des modèles permet d'individualiser trois zones principales (fig. 3) :

- une zone immobile située au dessus de la butée rigide nommée «domaine stable »;

- une zone caractérisée par des décrochements subparallèlles entre eux nommée «faisceau cisaillant»; 


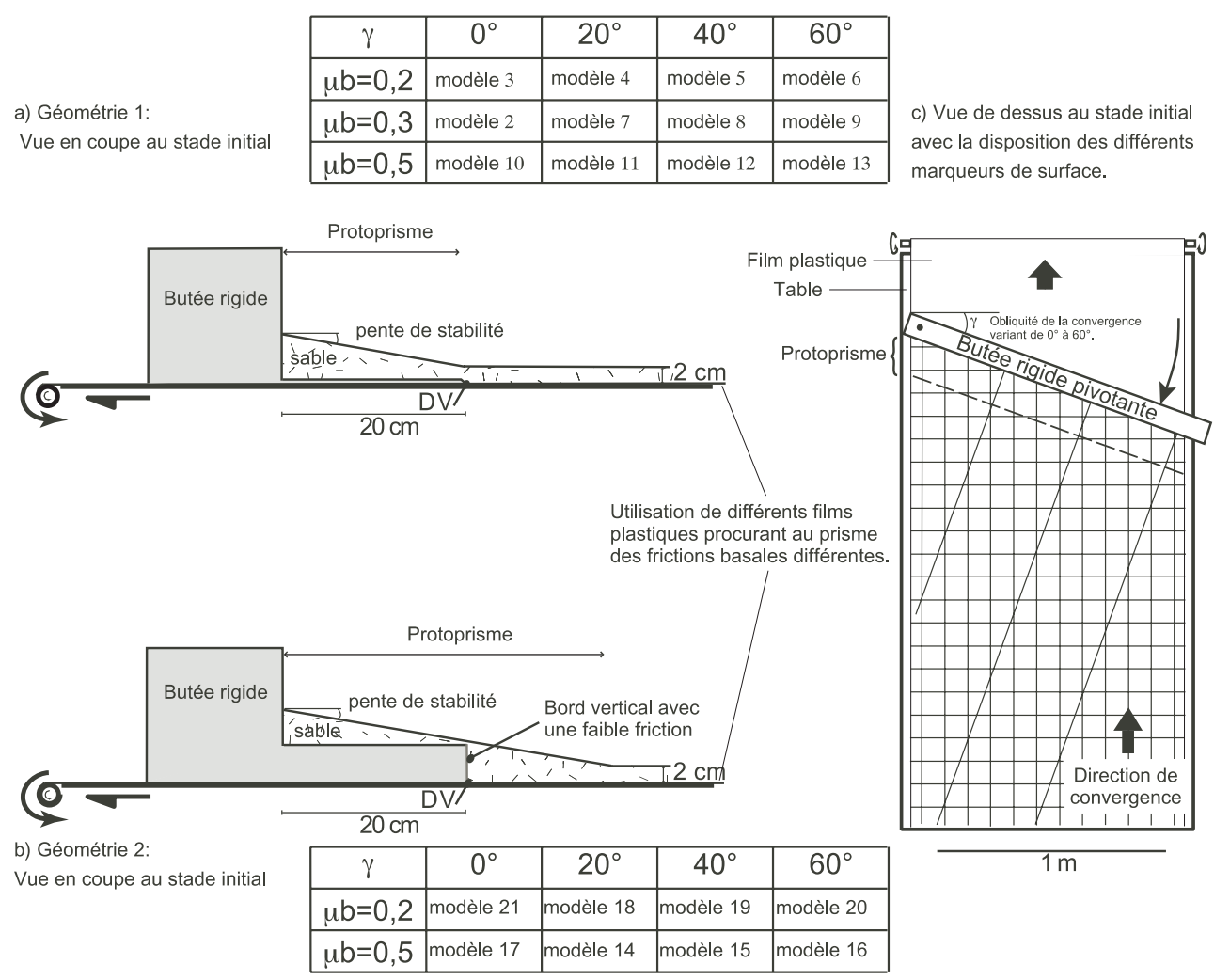

FIG. 1. - Experimental device.

FIG. 1. - Dispositif expérimental.

- une zone constituée d'unités accrétées nommée «prisme d'accrétion».

Le domaine stable existe dans tous les modèles et permet d'avoir un référentiel fixe au cours du raccourcissement.

\section{Géométrie 1 : discontinuité de vitesse sans bord vertical}

Le faisceau cisaillant se forme lorsqu'il y a obliquité de la convergence et quelle que soit la friction basale, excepté pour une forte obliquité $\left(\gamma=60^{\circ}\right)$ et une forte friction basale $(\mu \mathrm{b}=0,5)$ où seul le bourrelet existe. Cependant lorsque la friction est faible $(\mu b=0,2)$, le faisceau se constitue pour une obliquité de $60^{\circ}$. Il se forme pour des frictions basales moyennes et fortes $(\mu b=0,3$ et 0,5$)$ et des obliquités de $40^{\circ}$ et $60^{\circ}$. Ce faisceau et ses décrochements sont localisés à l'aplomb de la discontinuité de vitesse.

Des structures obliques associées aux décrochements sont observées et sont subperpendiculaires à la direction de convergence. Elles semblent dépendre du fonctionnement des décrochements.

Ces modèles se distinguent de la seconde série par une déformation diffuse.

\section{Géométrie 2 : discontinuité de vitesse avec bord vertical}

Dans cette série d'expériences, le faisceau est présent dans tous les modèles et n'est pas situé à l'aplomb de la discontinuité de vitesse. Il est décalé vers le front de déformation. Il est caractérisé par des failles décrochantes en échelon de longueurs équivalentes localisées à l'aplomb de la discontinuité de vitesse présentant un bord vertical. Pour une forte Bull. Soc. géol. Fr., 2002, $\mathrm{n}^{\mathrm{o}} 1$

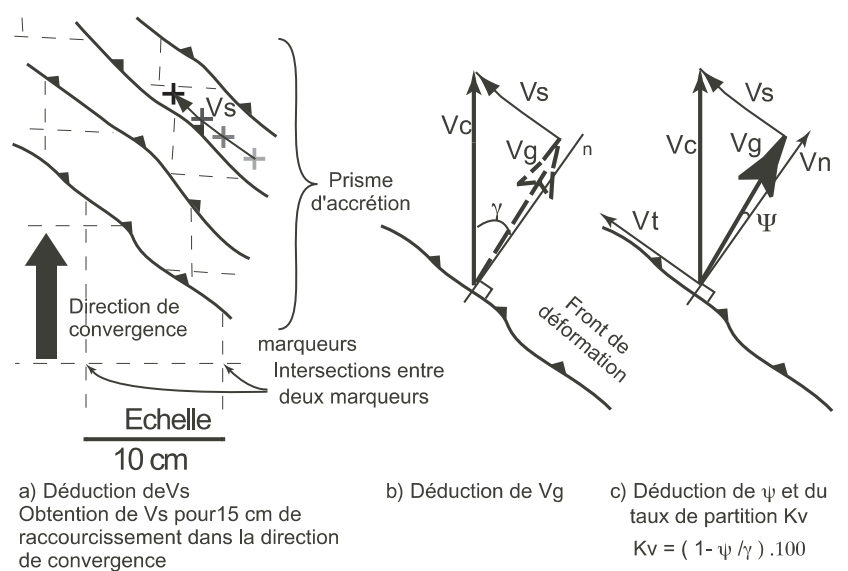

Vt: composante parallèle au front de déformation du vecteur convergence $\mathrm{Vn}$ : composante normale au front de déformation du vecteur convergence Vs: vecteur déplacement des points situés à la surface du prisme

$\checkmark c$ : vecteur convergence

$\mathrm{Vg}$ : vecteur glissement

FIG. 2. - Procedure to determine the slip vector Vg, its angle of obliquity $\psi$ with the perpendicular to the deformation front, and the partition rate $\mathrm{Kv}$ $(\%)$

FIG. 2. - Procédure de détermination du vecteur glissement $\mathrm{Vg}$ de son angle d'obliquité $\psi$ avec la normale au front de déformation ainsi que du taux de partition Kv (\%).

friction basale $(\mu b=0,5)$ et une forte obliquité $\left(\gamma=60^{\circ}\right)$, le faisceau incorpore les unités accrétées réduisant ainsi la largeur du prisme.

Des structures obliques associées aux décrochements apparaissent au cours du raccourcissement. Elles sont sub- 


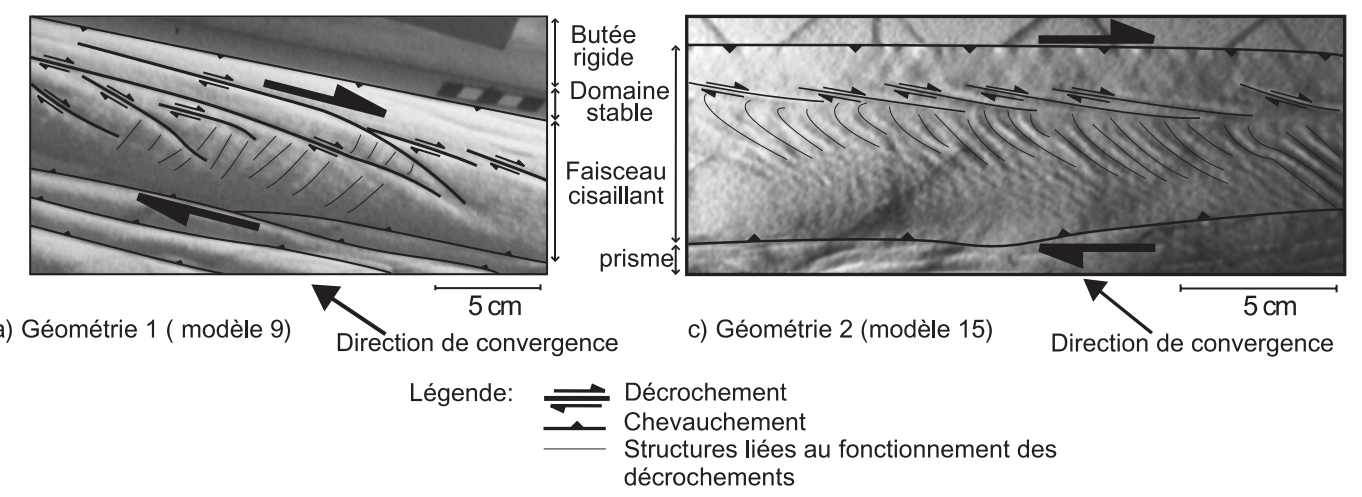

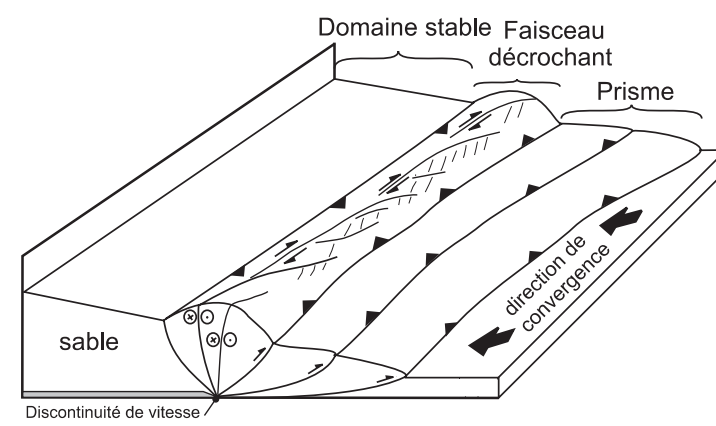

b) schéma caractérisant les modèles de géométrie 1

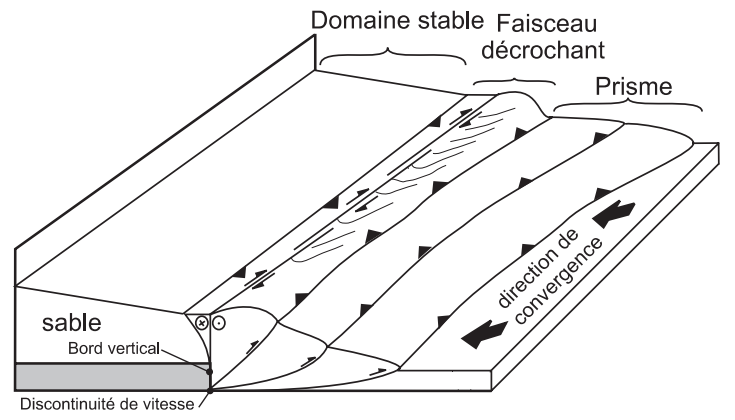

d) schéma caractérisant les modèles de géométrie 2

FIG. 3. - Relation between structures associated with strike slip faults for both backstop geometries used : (a) geometry 1 , (c) geometry 2 and the diagrams (b and c) characterizing each models serie. We note that these structures have different orientations with respect to the backstop geometry. FIG. 3. - Relation entre les structures associées aux décrochements suivant les deux géométries de butées rigides utilisées : (a) géométrie 1, (c) géométrie 2 et schémas (b et d) caractérisant chaque série de modèles. On remarque que ces structures ont des orientations différentes en fonction de la géométrie de la butée rigide.

parallèles à la direction de convergence et sont localisées entre les décrochements et la première unité accrétée du prisme.

Le prisme d'accrétion caractérise également dans cette seconde série les modèles de faible obliquité, quelle que soit la friction basale. Pour des obliquités plus fortes $\left(\gamma=40^{\circ}\right.$ et $\left.60^{\circ}\right)$, le prisme est d'autant plus réduit que la friction basale et l'obliquité sont élevés.

Ces modèles montrent par la position des décrochements à l'aplomb de la discontinuité de vitesse que la déformation est mieux localisée avec le second type de géométrie.

On note que ces trois zones s'individualisent au delà d'un seuil d'obliquité. Il est franchi pour une obliquité de convergence supérieure ou égale à $40^{\circ}$ pour des frictions basales moyennes et fortes $(\mu b=0,3$ et 0,5$)$. Pour une friction basale faible $(\mu b=0,2)$, il est franchi pour une obliquité de convergence supérieure ou égale à $60^{\circ}$.

Ensuite, à chaque stade final des modèles, des mesures sont effectuées sur les pentes de stabilité du prisme. Leur analyse sur les deux séries de modèles montre que $\alpha c$ et $\alpha$ n augmentent avec la friction basale et que on augmente avec l'obliquité de la convergence, alors que $\alpha c$ reste à peu près constante lorsque l'obliquité croît (fig. 4). Des modèles expérimentaux de prismes d'accrétion en convergence frontale indiquent également que la pente de stabilité du prisme augmente avec la friction basale [Malavieille et al., 1992]. Le sable sec utilisé dans les expériences comme analogue des matériaux du prisme obéit au critère de rupture de Coulomb. Davis et al. [1983] ont appliqué ce critère à la stabili-
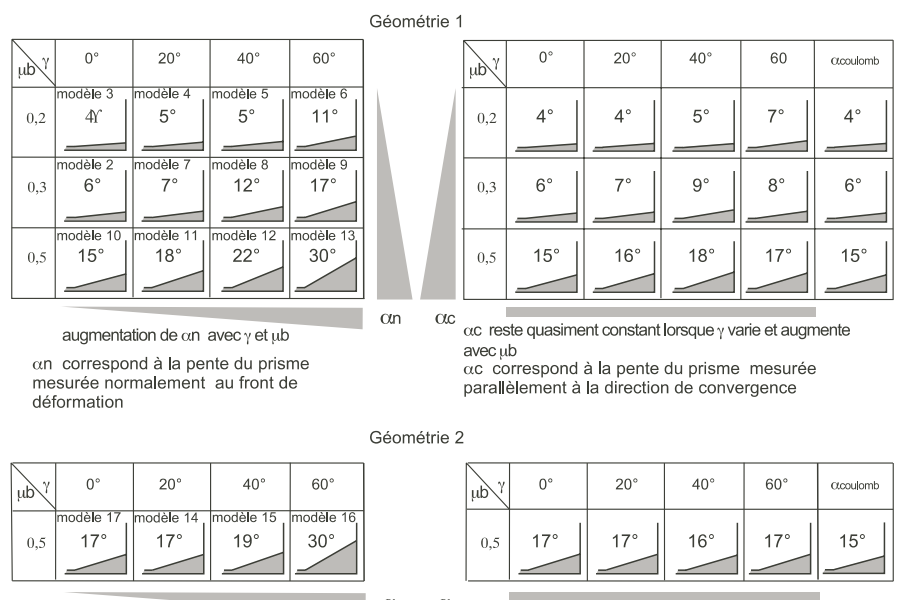

Géométrie 2

augmentation de $\alpha$ avec $\gamma$

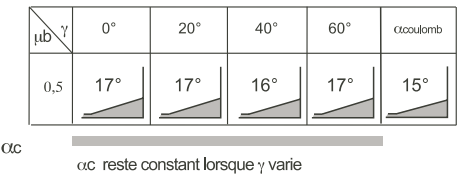

FIG. 4. - Evolution of $\alpha$ n and $\alpha c$ with $\gamma$ and $\mu$ b for both backstop geometries types. We note that $\alpha_{\text {coulomb }}$ is around equal to $\alpha \mathrm{c}$. All measurements accurate to within $2^{\circ}$

FIG. 4. - Evolution des pentes $\alpha \mathrm{n}$ et $\alpha \mathrm{c}$ avec g et $\mu \mathrm{b}$ pour les deux types de géométrie de butée rigide. On remarque que $\alpha_{\text {coulomb }}$ est à peu près égal à ac. Toutes les mesures sont effectuées à $2^{\circ}$ près.

té des prismes convergents et ont obtenu la relation suivante lorsque la convergence est normale :

$$
\alpha_{\text {coulomb }}+\gamma=\frac{(\mu b+\beta)}{(1+K)}
$$


avec $\alpha_{\text {coulomb }}$ : pente de stabilité $; \beta$ : pente de la plaque plongeante $; \mu \mathrm{b}$ : friction basale $; \mathrm{K}$ : paramètre fonction du coefficient de friction basal et interne $\mu \mathrm{b}$ et $\mu$.

Le taux de partition $K_{V}$ est fonction du rapport entre le vecteur convergence $V_{n}$ et le vecteur glissement $V_{g}$. Chacun peut être défini par son obliquité par rapport à la normale à la fosse qui sont respectivement les angles $\gamma$ et $\psi$. Le calcul du taux de partition selon Liu et al. [1995] est donné par l'équation (1), avec $\gamma$ : angle d'obliquité du vecteur convergence; $\psi$ : angle d'obliquité du vecteur glissement

Pour les deux géométries de butée rigide, on remarque (fig. 5) que : le taux de partition $\mathrm{K}_{\mathrm{V}}$ augmente avec l'obliquité de la convergence $\gamma$, et la partition du mouvement existe pour $\gamma$ supérieur ou égal à $20^{\circ}$. Cependant aucun modèle n'a été réalisé entre $0^{\circ}$ et $20^{\circ}$, on ne peut donc pas savoir définitivement si une partition se produit pour $\gamma$ inférieur à $20^{\circ}$.

Dans la seconde série d'expériences, le taux de partition est homogène dans toute la structure. Ceci confirme que la déformation est localisée, contrairement à la première série d'expériences où elle semble plus diffuse. Toute la structure se déplace en bloc le long d'un décrochement situé à l'aplomb de la discontinuité de vitesse à l'arrière du prisme d'accrétion.

Effectivement, on peut voir sur le modèle 6 (fig. 6), que les vecteurs glissement (représentés par les grosses flèches) des points en surface n'ont pas une orientation équivalente le long de la structure ainsi qu'à différents stades de raccourcissement. La longueur des flèches n'est pas toujours identique. Ceci est dû à la disparition du point d'intersection des marqueurs lors du raccourcissement. Le vecteur $\mathrm{V}_{\mathrm{g}}$ n'est pas calculé de ce fait pour le même taux de raccourcissement suivant les points étudiés. Dans le modèle 20, on remarque par contre que l'orientation des vecteurs glissement est semblable sur toute la structure et ne varie pas au cours du raccourcissement.

\section{DISCUSSION}

L'étude de la stabilité des prismes d'accrétion a permis à Davis et al. [1983] d'obtenir une relation qui s'applique dans le cas d'une convergence frontale, dans la direction de convergence. Dans les expériences réalisées ici, les mesures effectuées montrent que la pente de stabilité du prisme d'accrétion, lorsqu'on la mesure dans la direction de la convergence, est sensiblement égale à $\alpha_{\text {coulomb }}$ et reste constante. Par contre celle-ci augmente dans la direction perpendiculaire au front de déformation. L'équation (2) semble donc s'appliquer uniquement dans la direction de convergence. Ainsi, on ne peut déduire des paramètres fiables à partir de mesures sur le prisme seulement si celles-ci sont effectuées dans la direction de la convergence (même si elles semblaient plus logique dans la direction de convergence relative, c'est-à-dire suivant $\mathrm{V}_{\mathrm{g}}$ ).

Pinet et Cobbold [1992], dans leurs modèles analogiques constitués d'une couche de silicone (niveau ductile) sur laquelle repose du sable représentant le niveau fragile, ainsi que McCaffrey [1992], indiquent l'existence d'un seuil d'obliquité de $30^{\circ}$. Nos expériences, réalisées uniquement avec du sable, montrent que la partition du mouvement se produit non seulement pour de faibles obliquités de convergence, qu'elle augmente avec elle, mais aussi, Bull. Soc. géol. Fr., 2002, $\mathrm{n}^{\mathrm{o}} 1$
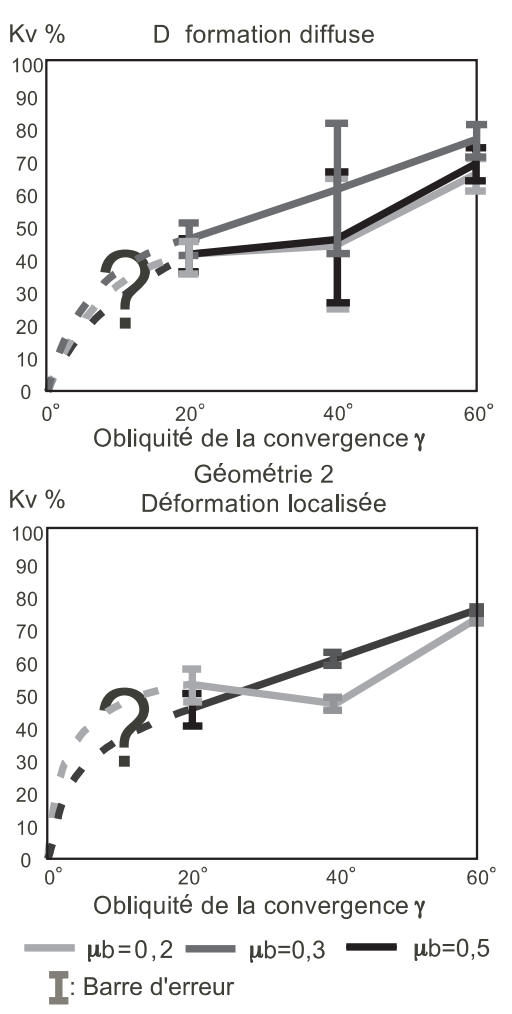

FIG. 5. - Partition rate versus the obliquity of convergence for both geometries with different basal friction $\mu \mathrm{b}$.

FIG. 5. - Taux de partition en fonction de l'obliquité de la convergence pour les deux géométries de discontinuités de vitesse utilisées avec des frictions basales $\mu \mathrm{b}$ différentes.

qu'elle apparaît pour une faible friction basale. Chemenda et al. [2000] montrent dans des modèles d'échelle lithosphérique, que la partition du mouvement est possible avec une forte friction interplaque. Les propriétés rhéologiques du matériau auraient donc une forte influence sur le seuil d'obliquité, au delà duquel la partition du mouvement se produit [Platt, 1993].

On note aussi que la géométrie de la butée n'influe en rien sur la partition du mouvement (celle-ci a lieu pour les deux géométries testées). Par contre, elle joue un rôle déterminant sur la localisation de la déformation (le décrochement est situé à l'arrière de la zone de subduction dans le cas de la géométrie de butée rigide 2 présentant un bord vertical de $4 \mathrm{~cm}$ ).

\section{Comparaison avec des exemples naturels}

Au sud de la fosse des Ryukyus, au nord-est de Taiwan, les données bathymétriques multifaisceaux mettent en évidence un décrochement dextre en arrière du prisme d'accrétion [Lallemand et al., 1999] très bien localisé. En parallèle, des études de sismique réflection et réfraction ont permis de mieux caractériser la géométrie de la terminaison du socle chevauchant [Font et al., 2001] et suggère que celui-ci se termine en formant un mur subvertical de plusieurs kilomètres de haut à l'aplomb du décrochement majeur. Les résultats obtenus dans les modèles analogiques de la seconde série (géométrie 2 : bord vertical de $4 \mathrm{~cm}$ ) confortent l'hypothèse que la terminaison du socle de la marge du Sud des Ryukyus est constituée d'un haut mur subvertical qui localise la partition de la déformation. 

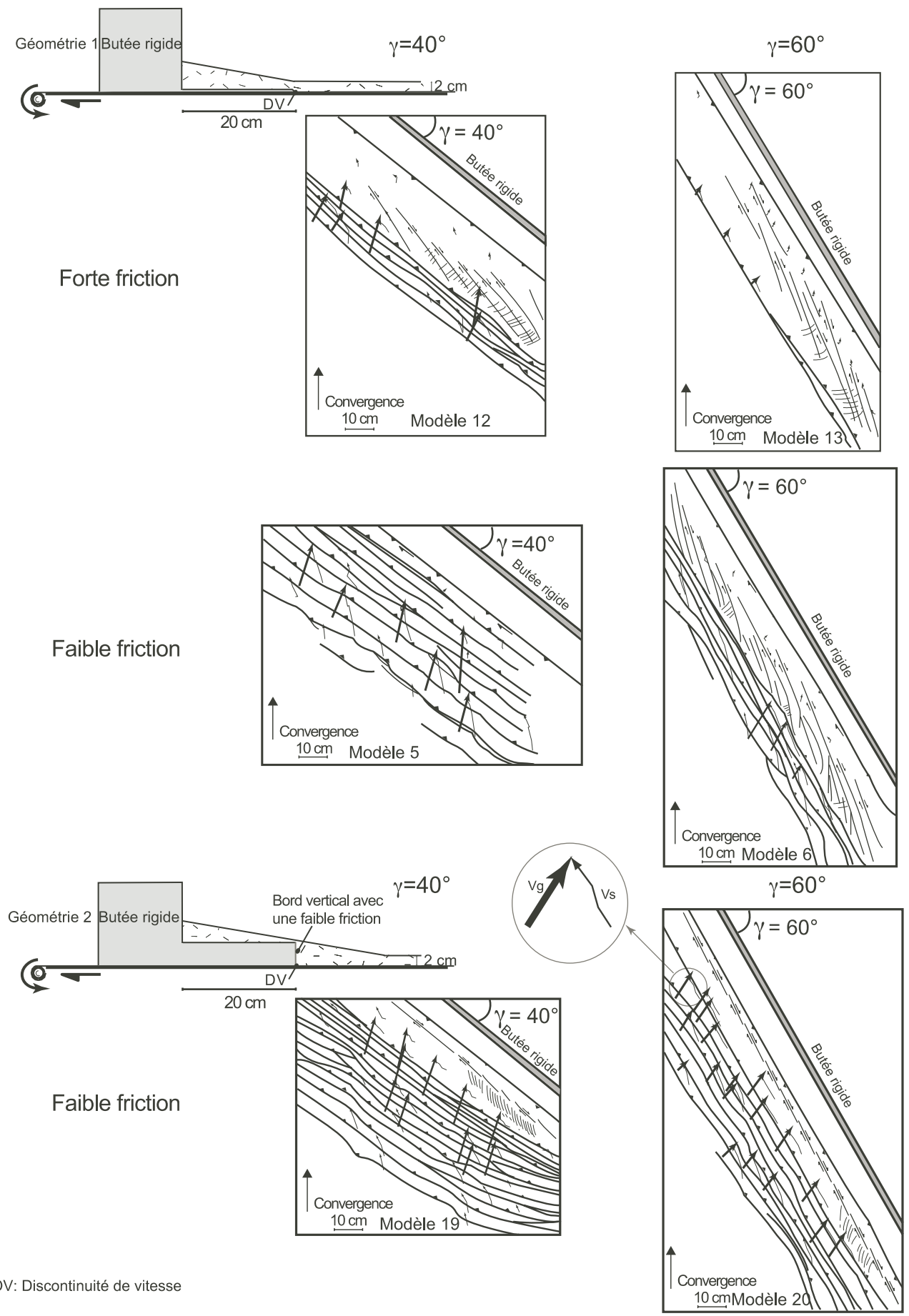

DV: Discontinuité de vitesse

FIG. 6. - Models showing three variable parameters : obliquity $\gamma$, basal friction $\mu \mathrm{b}$, backstop geometry. The location of the deformation is showed by the homogeneity of directions of slip vector in the second series of experiments (geometry2, models 19 and 20) in comparison with the first where slip vector have any common direction.

FIG. 6. - Modèles montrant les trois paramètres variables : l'obliquité $\gamma$, la friction basale $\mu \mathrm{b}$, la géométrie de butée rigide. La localisation de la déformation est montrée par l'homogénéité des directions des vecteurs glissement dans la seconde série d'expériences (géométrie 2 , modèles 19 et 20$)$ par rapport à la première où les vecteurs n'ont pas de directions communes.

En Nouvelle-Zélande, la plaque pacifique converge de façon très oblique $\left(60^{\circ}\right)$ au niveau de la marge d'Hikurangi. Entre le détroit de Cook $\left(175^{\circ} 00 \mathrm{E}\right.$ et $\left.176^{\circ} 45 \mathrm{E}\right)$, trois failles crustales de même direction sont localisées. La faille de Palliser-Kaiwhata qui souligne le rebord extérieur continental est interprétée comme une faille décrochante subverticale. Les deux autres, plus proches de la fosse, sont interprétées comme des failles inverses avec une composante décrochante oblique possible. Les données bathymé- triques multifaisceaux révèlent des linéaments associés à la trace de la faille de Palliser-Kaiwhata seulement [Barnes et al., 1998]. Dans nos modèles expérimentaux, des structures obliques associées au décrochement sont présentes (cas de la géométrie de butée 2). Celles-ci semblent dépendre du fonctionnement décrochant des failles. Connaissant les conditions aux limites de nos modèles et la surface structurale d'une partie du prisme d'Hikurangi, une configuration semblable à nos modèles pourrait exister. Elle serait carac- 
térisée par la présence d'une discontinuité de vitesse à l'aplomb de la faille de Palliser-Kaiwhata.

A Sumatra, Bellier et al. [1999], ont réussi à mieux contraindre les déplacements le long de la faille lithosphérique de Sumatra. Ils suggèrent que pour des obliquités de convergence faibles $\left(20^{\circ}\right)$, la partition du mouvement peut exister. Nos modèles montrent que la partition du mouvement existe pour des obliquités de convergence supérieures ou égales à $20^{\circ}$ ce qui conforte leur observation de partition du mouvement pour de faibles obliquités de convergence.

Dans les Andes du Nord, Ego et al. [1996] ont étudié les mécanismes au foyer des séismes, apportant des informations sur la déformation de différentes zones. Ces travaux suggèrent que lorsque l'obliquité de convergence est grande, des décrochements sont initiés plus facilement, alors que lorsqu'elle est faible, le raccourcissement est dominant. La déformation est accommodée dans nos modèles à l'arrière du prisme d'accrétion, par des décrochements de plus en plus nombreux lorsque l'obliquité de la convergence augmente.

\section{CONCLUSION}

Tout d'abord, la modélisation montre que la pente de stabilité du prisme d'accrétion reste à peu près constante dans la direction de convergence lorsque l'obliquité augmente. Sa valeur est équivalente à celle de la pente d'équilibre déterminée par la théorie du prisme de Coulomb.

On constate que le taux de partition du mouvement augmente avec l'obliquité de la convergence, ceci quelle que soit la friction basale du modèle. D'autres études portant sur des systèmes bicouches de rhéologie différentes indiquent un seuil d'obliquité pour la partition. La rhéologie des matériaux utilisés semble influencer le seuil de partition du mouvement.

Enfin, on observe trois domaines structuraux distincts qui s'individualisent au delà d'un seuil d'obliquité, ainsi que la présence de structures obliques associées aux décrochements. Ces structures pourraient permettre, de manière indirecte, de mieux caractériser la géométrie de la terminaison du socle de la marge continentale, qu'il n'est pas toujours aisé de déterminer dans les zones de subduction à partir des seules données de géophysique.

\section{Références}

Barnes P.M., Mercier De Lepinay B., Collot J.y, Delteil J. \& Audru J.C. (1998). - Strain partitioning in the transition area between oblique subduction and continental collision, Hikurangi margin, New Zealand. - Tectonics, 17, 4, 534-557.

Barrier E., Huchon P. \& Aurelio M. (1991). - Philippine fault : a key for Philippine kinematics. - Geology, 19, 32-35

Bellier O., Sebrier M. \& Pramumijoyo S. (1991). - La grande faille de Sumatra : géométrie, cinématique et quantité de déplacement mises en évidence par l'imagerie satellitaire. - C. R. Acad. Sci. Paris, 312, 1219-1226.

Bellier 0. \& Sebrier M. (1995). - Is the slip rate on the Great Sumatran Fault accomodated by fore-arc streching. - Geophys. Res. Lett., 22, 15, 1969-1972.

Bellier O., Bellon H., Sebrier M., Sutanto \& Maury R.C. (1999). $\mathrm{K}-\mathrm{Ar}$ age of the Ranau Tuffs : implications for the Ranau caldera emplacement and slip-partitionning in Sumatra (Indonesia). Tectonophysics, 302, 347-359.

Chemenda A., Lallemand S. \& Bokun A. (2000). - Strain partitionning and interplate friction in oblique subduction zones : constraints provided by physical modeling. - J. Geophys. Res., 105, 3, 5567-5582.

Dalhen F.A. \& Suppe J. (1984). - Mechanics of fold and thrust belts and accretionnary wedges: codes Coulomb theory. - J. Geophys. Res., 89, 10087-10101.

Davis D., Suppe J. \& Dalhen F.A. (1983). - Mechanics of fold- and-thrust belts and accretionary wedges. - J. Geophys. Res., 88, B2,1153-1172.

Dominguez S., Lallemand S., Malavieille J. \& Schnurle P. (1998). Oblique subduction of the Gagua ridge beneath the Ryukyu accretionnary wedge system insights from marine observations and sandbox experiments. - Mar. Geophys. Res., 20, 5, 383-402.

Ego F., Sebrier M., Lavenu A., Yepes H. \& Egues A. (1996). - Quaternary state of stress in the northern Andes and the restraining bend model for the Ecuadorian Andes. - Tectonophysics, 259, 101-116.
Font Y., Char-Shine LiU, Schnurle P. \& Lallemand S. (2001). - Constraints on backstop geometry of the Southwest Ryukyu Subduction based on reflection seismic data. - Tectonophysics, 333, $1-2,135-158$.

Lallemand S., Liu C-S., Dominguez S., Schnurle P., Malavieille J. \& the ACT scientific crew (1999). - Trench parallel stretching and folding of forearc basins and lateral migration of the accretionary wedge in the southern Ryukyus : a case of strain partition caused by oblique convergence. - Tectonics, 18, 2, 231-247.

Lallemand S. (1999). - La subduction océanique. - Gordon and Breach, Paris, 194 pages.

Liu X., McNally K.C. \& Shen Z.K. (1995). - Evidence for a role of the down going slab in earthquake slip partitioning at oblique subduction zones. - J. Geophys. Res., 100, 15,351-15,372.

Malavieille J., Calassou S., Lallemand S. \& Larroque C. (1992). Modélisation analogique des prismes d'accrétion océanique, film vidéo VHS de 28 minutes, produit et réalisé par la $\operatorname{SNEA}(\mathrm{P})$, série cours.

Malod J.A., Kemal B.M., Beslier M.O., Deplus C., Diament M., Karta K., Mauffret A., Patriat P., Pubellier M., Regnault H., Aritonang P., ZEN Jr., M.T. (1993). - Déformations du bassins d'avant-arc au Nord-Ouest de Sumatra : une réponse à la subduction oblique. - C. R. Acad. Sci., Paris, 316, 791-797.

McCAFFrey R. (1992). - Oblique plate convergence, slip vectors, and forearc deformation. - J. Geophys. Res., 97, 8905-8915.

McCAFFrey R. (1996). - Slip partitioning at convergent plate boundaries of SE Asia. - Geol. Soc. Spec. Publ., 106, 3-18.

McCAFFREY R. (1996). - Estimates of arc-parallel strain rates in fore arcs. - Geology, 24, 27-30.

Pinet N. \& Cobbold P.R. (1992). - Experimental insights into the partitioning of motion within zones of oblique subduction. - Tectonophysics, 206, 371-388.

Platt J.P. (1993). - Mechanics of oblique convergence. - J. Geophys. Res., 98, 9, 16239-16256. 